\title{
Resilience of Patients With Chronic Physical Diseases: A Systematic Review and Meta-Analysis
}

\author{
Reza Ghanei Gheshlagh, ${ }^{1}$ Kourosh Sayehmiri, ${ }^{2}$ Abbas Ebadi, ${ }^{3}$ Asghar Dalvandi, ${ }^{4}$ Sahar Dalvand, ${ }^{5}$ and \\ Kian Nourozi Tabrizi ${ }^{4, *}$ \\ ${ }^{1}$ PhD Candidate in Nursing Education, Department of Nursing, University of Social Welfare and Rehabilitation Sciences, Tehran, IR Iran \\ ${ }^{2}$ Associate Professor, Department of Biostatistics, Prevention of Psychosocial Injuries Research Center, Ilam University of Medical Sciences, Ilam, IR Iran \\ ${ }^{3}$ Associate Professor, Behavioral Sciences Research Center, Nursing Faculty, Baqiyatallah University of Medical Sciences, Tehran, IR Iran \\ ${ }^{4}$ Associate Professor, Department of Nursing, University of Social Welfare and Rehabilitation Sciences (USWR), Tehran, IR Iran \\ ${ }^{5}$ MSc in Biostatics, Social Determinants of Health Research Center, Kurdistan University of Medical Sciences, Sanandaj, IR Iran \\ "Corresponding author: Kian Nourozi Tabrizi, Assistant Professor, Department of Nursing, University of Social Welfare and Rehabilitation Sciences (USWR), Tehran, IR Iran. Tel: \\ +98-9122073826, Fax: +98-2122180036, E-mail: Dr.kian_nourozi@yahoo.com
}

Received 2016 April 17; Revised 2016 May 24; Accepted 2016 June 20.

\begin{abstract}
Context: Resilience can be seen as an adaption to stress, such as that caused by health problems or disease, that attenuates the negative effects of stress. The present research performed a systematic review and meta-analysis to study resilience scores among adults diagnosed with chronic physical diseases.

Evidence Acquisition: Electronic databases, including Persian language (scientific information database [SID], IranMedex, Magiran, IranDoc, and Medlib) and English language (Google Scholar, Science Direct, PubMed, Pre-Quest, and Scopus), were searched. Fifteen articles were found using the keywords disease or chronic disease, resilience or resiliency, and illness, either alone or in combination, both in Persian and English languages. Data analysis was carried out through meta-analysis (random-effects model), and heterogeneity was investigated by subgroup and meta-regression analyses. The data were analyzed in STAT software (12.0).

Results: The mean resilience score of the chronic disease patients $(n=3369)$ was 74.6 (95\% CI: 51.8 - 97.4). In terms of diseases, the mean resilience score of cancer patients was 79.6 (95\% CI: $48.3-111.1)$, whereas it was 79.6 for cardiovascular disease patients (95\% CI: 45.8 - 113.3) and 64.6 for patients with other diseases (95\% CI: 6.6 - 122.7). There was no relationship between the resilience of chronic disease patients and the year of the study $(\mathrm{P}=0.711)$ and the sample size in the studies $(\mathrm{P}=0.351)$.

Conclusions: The mean resilience score of the patients was less than that of healthy individuals. As resilience can be acquired at any stage of life, irrespective of age and disease status, there is a need for training to improve resilience among patients through educational programs.
\end{abstract}

Keywords: Resilience, Chronic Disease, Meta-Analysis

\section{Context}

Chronic diseases adversely affect all physical, mental, and social aspects of a person's life, as they are long term, incurable without intervention, and cannot be cured completely $(1,2)$. Chronic diseases are undeniable stressors, as they threaten welfare, physical integrity, future plans, and financial stability and undermine the ability to fulfill family, social, and professional roles (3). Patients diagnosed with chronic diseases encounter new situations for which usual resistance strategies may not be effective and call for new strategies to be adopted (4). Although these responses and reactions are normal at first and even helpful in adapting to the new situation, if the problem is not approached properly, it influences the whole patient's life (5). There are different ways of dealing with the problems and stress associated with chronic diseases (6). Although people tend to show purposeful responses to stres- sors, there is no guarantee these responses are the best possible ones (7). Resilience is one way to respond to stress, allowing the individual to adapt to various stressors, such as injuries, threats, tragic events, interpersonal and family problems, financial problems, work- and health-related problems, and diseases. The aim of resilience is to reduce the negative effects of the stressor $(8,9)$.

There is no general definition of resilience. However, there is agreement among authors about some of the main features of resilience, for example, returning to normal performance, increasing adaptability, and preserving mental health $(10,11)$. Resilience is not a general structure for all life areas, but it is rather a personal, cultural, dynamic, and background-dependent phenomenon. People may not demonstrate resilience to all life events or aspects but only to specific situations. That is, people may be resilient to specific threats and vulnerable to others (12- 
14). In addition, some sources of resilience are only achievable in specific contexts; which means it is a way of interaction between stressors, context, and personal traits (15). For example, resilient individuals may encounter harmful situations, but they do not suffer interruption of their life order (16). Studies showed that resilience in patients with chronic physical diseases was associated with various factors, such as self-care, adhering to treatment programs, health-related life quality, patients' perceptions of the disease and pain, adherence to physical activity, selfempowerment, increasing self-efficiency, reducing depression, anxiety, and stress, increasing optimistic viewpoint, and accelerating recovery (17-21). Most studies in the literature on resilience have focused on subjects who had sustained severe trauma and had social problems or poor children who had been victims of physical abuse. However, there is a paucity of studies on chronic disease patients (22, 23).

Vanderbit-Adriance and Shaw (cited in Windle et al.) maintained that the resilience level ranged from 25\% - 84\%. This wide range of resilience problematizes the comparison of the results with other works or giving a clear-cut definition of resilience even when the subjects suffer from similar problems. Another question to be asked is how to measure this concept in different experiences. In a study of resilience tools, Windle et al. maintained that no existing tools are psychometerically standard (24). Based on the aforementioned issues, the present research aimed to study resilience among chronic disease patients to demonstrate the need to design specific tools.

\section{Evidence Acquisition}

\subsection{Data Source}

We performed a systematic review of the literature and a structured meta-analysis to compare the resilience of patients with chronic physical diseases based on the type of disease, tools, place of study, and type of study. To this end, all published articles in Farsi and English between 2000 and May 2016 in this field were reviewed systematically, according to PRISMA guidelines (25).

\subsection{Search Strategy}

The articles were selected by searching national and international databases, such as Google Scholar, Science Direct, PubMed, Pre-Quest, Scopus, scientific information database (SID), IranMedex, Magiran, IranDoc and Medlib. The search keywords were: resilience or resiliency, illness, and disease or chronic disease, either alone or in combination, both in Persian and English languages. In the case of Persian articles, the Persian equivalents of the terms were used. To extend the search area, the references in the articles were checked, and a further search was performed based on the type of the prevalent diseases.

\subsection{Data Collection}

First, all articles on resilience among adults with chronic diseases were selected. The inclusion criteria were as follows: published in Persian or English; interventional or observational studies, with a quantitative analytical component; availability of full text; published in the last 16 years (2000 - 016); and adults ( $>18$ years) with chronic physical diseases as the study group. Studies on children and psychological-mental patients were excluded, as well as studies with insufficient data, gray literature (dissertations, case reports, reviews, editorials, animal studies, and conference representations), studies on specific symptoms of chronic diseases, such as inability and chronic pain, and studies that did not utilize resilience tools. Two researchers (RGG and $\mathrm{AE}$ ) screened the articles independently twice: once based on the titles and abstracts and once based on the full texts.

A specially designed form was used to extract data from the articles. The form listed the author(s)' name(s), year of publication, place of publication, type of study, sample group size, data gathering tool, and resilience score. To reduce bias, the authors were blinded to the details of the articles, including the names of the authors and magazines. Two independent researchers performed the data extraction. In the case of disagreement, a second researcher (KS) made the final decision.

Two researchers (AD and KNT) examined the quality of the studies, using the quality of Life index, and disagreements, if any, were resolved by a second researcher, (KS). The quality of the studies was assessed according to study design parameters, samples, recording processes, control groups, number of participants, and psychometric measures. Each parameter was assigned a score of $0-3$, with the total score ranging from $0-15$ (26). Of 764 articles found in the primary search, 18 were entered in the study. Of these 18 articles, three articles were excluded because they used a combination of tools to assess resilience rather than one of the 15 available tools. Consequently, 15 articles were included in the final analysis (Figure 1).

\subsection{Statistical Analysis}

In this meta-analysis, taking into account that the stress score had a normal distribution, the variance of each study was estimated with respect to the normal distribution, as follows:

$$
\operatorname{Var}(\bar{x})=\frac{\sigma^{2}}{n}
$$




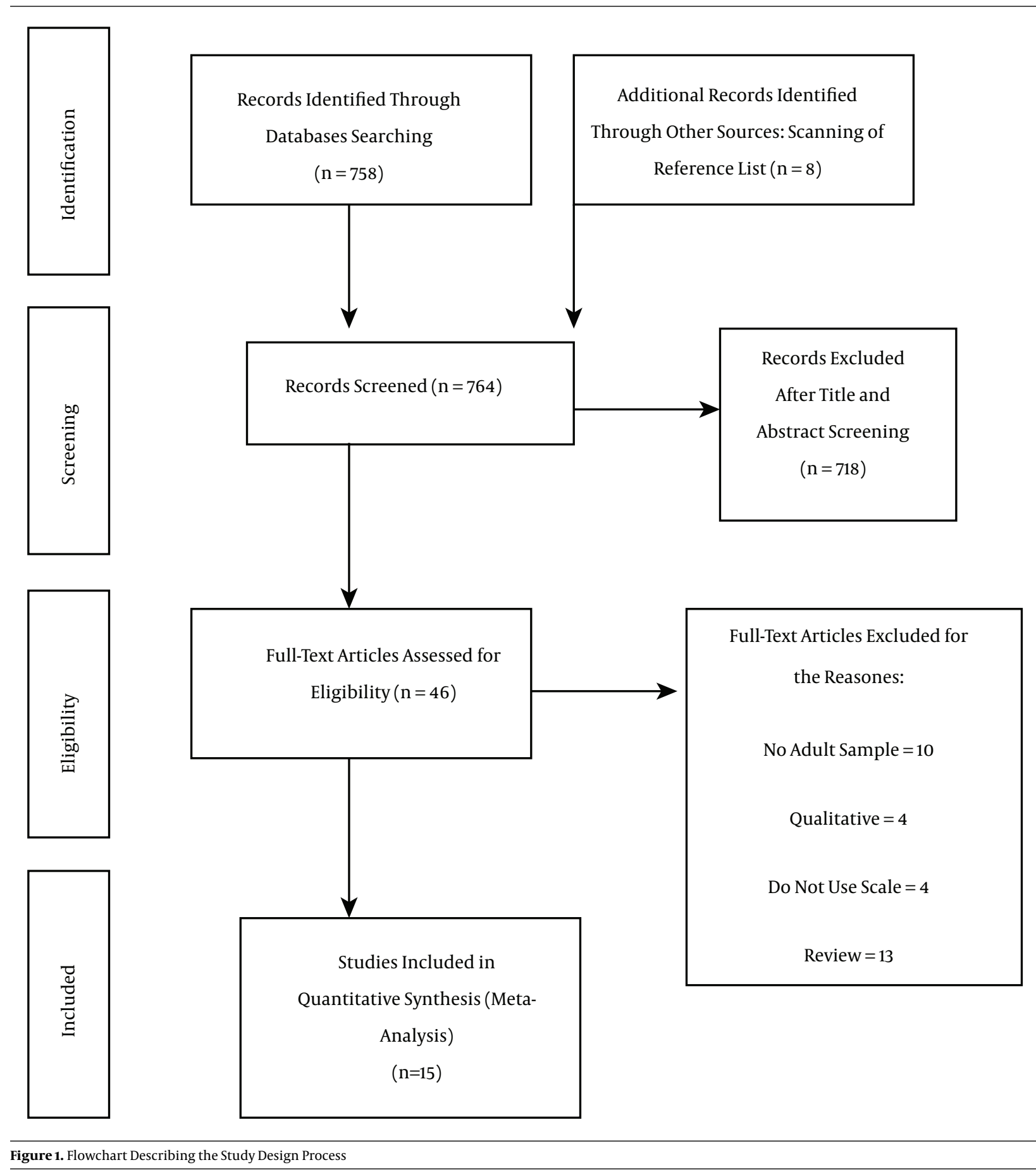

The weight of each study was relative to its inverse variance. A forest plot was used to visualize the heterogeneity among the studies. The mean resilience score of the individual studies and the overall mean score are presented in forest plots. The heterogeneity among the studies was evaluated using the $\mathrm{I}^{2}$ index, which describes the percentage of variation across the studies. A value of $\mathrm{P}<0.1$ was considered significant. $\mathrm{I}^{2}$ values of $25 \%, 25 \%-75 \%$, and above $75 \%$ were considered low, medium, and high heterogeneity, respectively. For the purpose of the meta-analysis, the included studies were assumed to be random samples from a population. Thus, a random-effects model was employed. 
To explore the heterogeneity among the studies, subgroup analyses, with a meta-regression, were performed. The relationship between the resilience score, year of the study, and study sample size was examined by a meta-regression analysis. For the variables of each study, such as the type of tool, disease, and geographical region, we conducted subgroup analyses to examine the mean resilience score. To explore potential publication bias, Egger's regression test was used to assess the funnel plot. Data analysis was performed using the Statistical Software Package (STATA), version 12.0 .

\section{Results}

The primary search yielded 764 articles. In the first stage of screening, 749 articles (irrelevant or in a language other than Farsi or English) were excluded. The final study consisted of 15 articles. Twelve articles (75\%) were in English. Of the 15 articles, six involved patients with cardiovascular diseases, four were of cancer patients, two were of patients with spinal cord injuries, one focused on patients suffering from renal failure, one was on AIDS patients, and one was on patients with systematic lupus erythematosus.

Resilience was measured by RS-25 ( $\mathrm{n}=5$ articles), CDRISC 25 ( $n=6$ articles), and CD-RISC 10 ( $n=4$ articles). The articles had been published between 2013 and 2016 and consisted of a total of 3369 participants (an average of 225 participants per study). The findings of the sensitivity analysis indicated that in the absence of the individual studies (excluding the results of each study from the total analysis), there was no significant change in the total finding of mean score of resilience.

Using an adapted version of the quality of life index appraisal tool, the mean score of the methodological quality of the articles was 6.75. The sample sizes of the studies differed, with 9 (60\%) studies having more than 100 participants each. The larger and smaller sample groups were in Rosenberg $(n=1823)$ and Faria $(n=40$; systematic lupus erythematosus patients). Further details are listed in Table 1.

A random-effects model was used to analyze the resilience scores of the chronic disease patients This model assumes that the observed differences are rooted in differences in sampling methods and patients' scores. The model revealed a resilience score of 74.65 for 3369 patients (95\% CI: 49.77 - 96.99). The articles were classified based on the place of study (continent), resilience tool, and type of disease (Table 2, Figure 2)

The results of the meta-regression (Figures 3 and 4 ) indicated that there was no relation between the patients' mean resilience scores and the year of publication ( $\mathrm{P}=$ $0.711)$ or sample size $(P=0.351)$. Thus, an increase in the sample size and the publication year did not affect the resilience score.

Research tools were featured with different number of statements, which made the obtained scores incomparable. To compare the mean resilience scores obtained by three research tools, CD-RISC10 (72.5\%), CD-RISC25 (60.5\%), and RS25 (66.8\%), the scores were expressed as percentages. The highest score (98.6) was found in the European studies, and the lowest (27.5) was found in the Australian studies (95\% CI: 55.6 - 141.5 and 23.7 -31.3, respectively). The next highest score was obtained in the Asian studies.

As shown in Figure 2, the mean resilience score of the cancer patients was 79.6 (95\% CI: 48.2 - 111.1), and the mean score of the cardiovascular patients was 79. (95\% CI 45.8 113.3), which was higher than that of the patients with other diseases (64.6; 95\% CI: 6.6 - 122.7).

The results of Egger's test indicated that there was no publication bias.

\section{Conclusions}

Resilience refers to how people, irrespective of their age, deal with the hardships of life, such as disease and threats. It has drawn a great deal of attention in political and clinical areas because of its potential effects on wellbeing, welfare, and quality of life (24). There is no general agreement between authors regarding the nature of resilience, with some concluding that it is a process or an outcome of a process and others stating that it is a personality trait. The differences in the definitions lead to problems in measuring resilience (11). At present, there are 15 tools to assess resilience, of which only 10 can be used in clinical situations. Each of these tools has its own limitations. The RSA tool has a low response rate and nonrandom sampling, and the RS tool does not elaborate on details. The subscales of the RISC tool are not reliable, and they do not include features of resilience. READ, Ego Resilience, and YR-ADS cannot measure resilience over time. The Dispositional scale focuses only on hardiness and does not match with the dynamic process of resilience and does not include a factor analysis.

In a survey of resilience tools, Windle et al. asserted that none of the tools was standard in terms of psychometric standards (24). The concept of resilience seem to be too broad to be measured by just one scale. The mean scores measured using the CD-RISC 25, CD-RISC 10, and RS-25 tools were 60.5 (range of 25 -100), 29 (range of $10-40$ ), and 117 (range of 25-175), respectively. To compare the mean scores obtained using the above-mentioned tools, the scores were expressed as percentages. The scores of the patients with chronic diseases using the CD-RISC 25, CD-RISC 10, and RS25 tools were $72.5 \%, 60.5 \%$, and $66.8 \%$, respectively. A higher 
Table 1. Articles Entered in the Systematic and Meta-Analysis of the Resilience of Adults With Chronic Physical Diseases

\begin{tabular}{|c|c|c|c|c|c|c|c|c|}
\hline \multirow[t]{2}{*}{ No. } & \multirow[t]{2}{*}{ The First Author } & \multirow[t]{2}{*}{ Publication Year } & \multirow[t]{2}{*}{ Sample Size } & \multirow[t]{2}{*}{ Scale } & \multirow[t]{2}{*}{ Place } & \multirow[t]{2}{*}{ Resilience Score } & \multicolumn{2}{|c|}{ 95\% CI } \\
\hline & & & & & & & Upper & Lower \\
\hline $\mathbf{1}$ & Dubey (27) & 2015 & 68 & CD-RISC25 & Sweden & 74.40 & 77.39 & 71.41 \\
\hline 2 & $\operatorname{Liu}(28)$ & 2015 & 128 & RS-25 & Taiwan & 143.09 & 147.05 & 139.13 \\
\hline 3 & Rosenberg (29) & 2015 & 1823 & CD-RISC10 & U.S. & 31.40 & 31.70 & 31.10 \\
\hline 4 & Arrebola-Morreno (30) & 2014 & 134 & RS-25 & Spain & 46.30 & 49.88 & 42.72 \\
\hline 5 & Faria (31) & 2014 & 40 & RS-25 & Brazil & 99.83 & 108.41 & 91.25 \\
\hline 6 & Hayter (32) & 2014 & 97 & CD-RISC10 & Australia & 25.65 & 27.26 & 24.04 \\
\hline 7 & Schumacher (33) & 2014 & 75 & RS-25 & Germany & 143 & 147.26 & 138.75 \\
\hline 8 & Dale (34) & 2014 & 85 & CD-RISC10 & U.S. & 29.39 & 31.06 & 27.72 \\
\hline 9 & Doustrade Tousi (35) & 2014 & 145 & CD-RISC25 & Iran & 46.36 & 51.24 & 41.48 \\
\hline 10 & Noori Saeid (36) & 2014 & 184 & CD-RISC25 & Iran & 65.50 & 67.77 & 63.23 \\
\hline 11 & Amipour (37) & 2014 & 106 & CD-RISC25 & Iran & 45.60 & 48.13 & 43.07 \\
\hline 12 & $\mathrm{Ma}(38)$ & 2013 & 150 & RS-25 & Taiwan & 139.05 & 140.16 & 137.99 \\
\hline 13 & Kilic (19) & 2013 & 60 & CD-RISC10 & Australia & 29.53 & 31.35 & 27.71 \\
\hline 14 & Moe (39) & 2013 & 120 & RS-25 & Norway & 130.83 & 133.69 & 127.97 \\
\hline 15 & $\operatorname{Min}(40)$ & 2013 & 152 & CD-RISC25 & Korea & 70.21 & 71.21 & 69.21 \\
\hline
\end{tabular}

Table 2. Mean Resilience Scores of the Patients With Chronic Physical Diseases in all the Subgroups

\begin{tabular}{|c|c|c|c|c|c|c|}
\hline \multirow[t]{2}{*}{ Study Group } & \multirow[t]{2}{*}{ Articles } & \multirow[t]{2}{*}{ Sample Size } & \multirow[t]{2}{*}{ Mean Resilience Scores } & \multirow[t]{2}{*}{$\mathbf{9 5} \% \mathrm{CI}$} & \multicolumn{2}{|c|}{ Heterogeneity } \\
\hline & & & & & $I^{2} \%$ & $\mathbf{P}$ \\
\hline \multicolumn{7}{|l|}{ Research tool } \\
\hline RS-25 & 5 & 647 & 117 & $89.6-144.4$ & 99.8 & 0.0001 \\
\hline CD-RISC25 & 6 & 657 & 60.5 & $50.3-70.7$ & 99 & 0.0001 \\
\hline CD-RISC 10 & 4 & 2065 & 29 & $26.3-31.7$ & 94.5 & 0.0001 \\
\hline \multicolumn{7}{|l|}{ Continent } \\
\hline Asia & 6 & 867 & 84.9 & $48.9-120.9$ & 100 & 0.0001 \\
\hline Europe & 4 & 397 & 98.6 & $55.6-141.5$ & 99.8 & 0.0001 \\
\hline U.S. & 3 & 1948 & 50.7 & $39.6-61.7$ & 99.2 & 0.0001 \\
\hline Australia & 2 & 157 & 27.5 & $23.7-31.3$ & 89.8 & 0.002 \\
\hline \multicolumn{7}{|l|}{ Country } \\
\hline Iran & 3 & 435 & 52.55 & $37.9-67.1$ & 98.6 & 0.0001 \\
\hline Other & 12 & 2934 & 80.1 & $53.4-106.9$ & 100 & 0.0001 \\
\hline Mean total score & 15 & 3369 & 74.6 & $51.8-97.4$ & 100 & 0.0001 \\
\hline
\end{tabular}

score denotes greater resilience. Based on this, the patients suffering from chronic physical diseases obtained nearly three-quarters of resilience score in CD-RISC 10.

The mean resilience score of the cancer patients was 70.6 (CI 95\%: 48.2 - 111.1), whereas it was 79.6 (CI 95\%: 45.8 - 113.3) for cardiovascular disease patients and 67.5 for pa- tients with other diseases (67.5; CI 95\%: 19.1 - 115.8). The results suggest that apparently, the more lethal the disease, the higher the patients' resilience to reduce the negative effects of the disease. Resilience, together with feelings of control and capability, make the patient feel that he/she has control over the disease and that he/she is capable of 


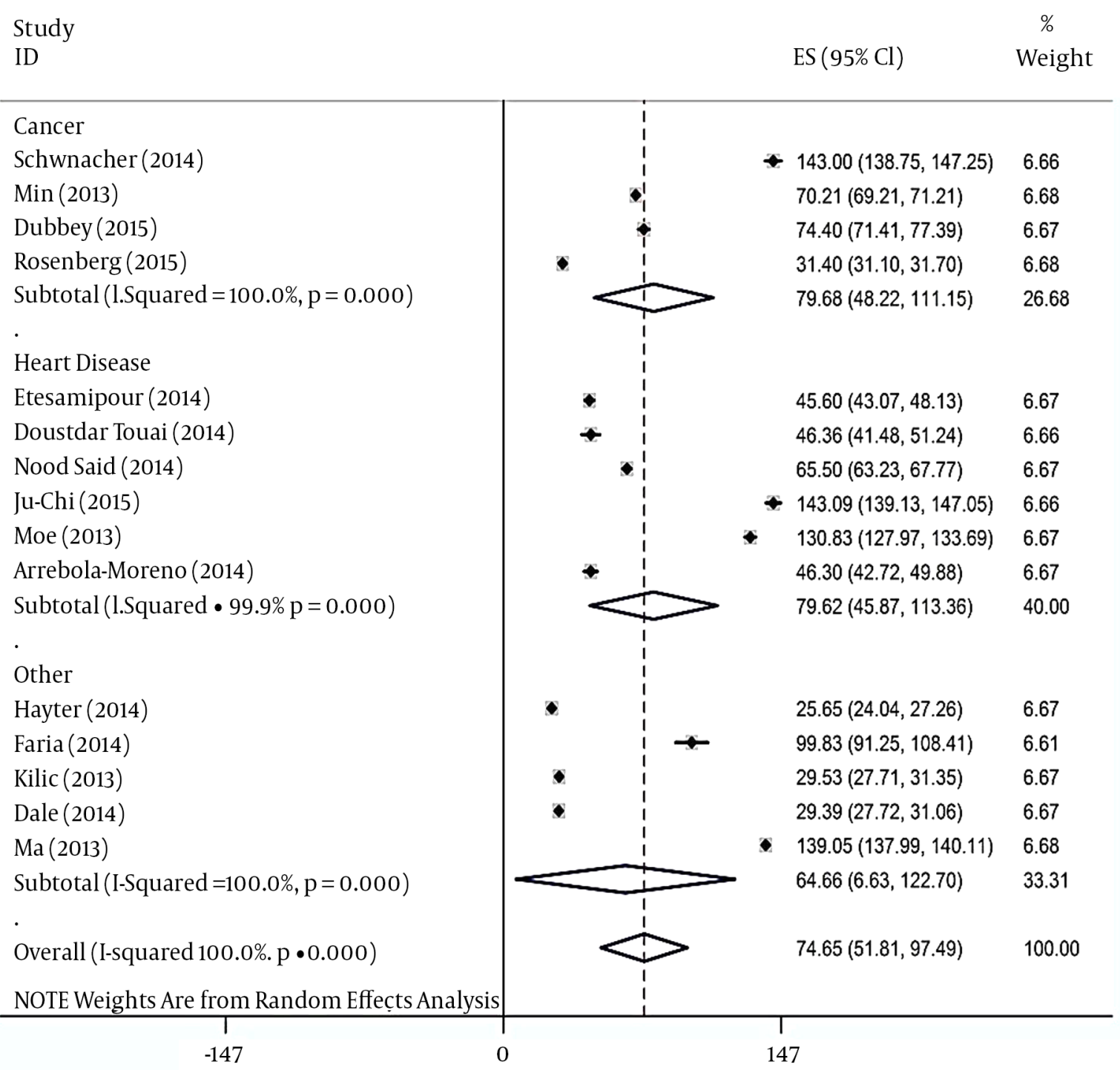

Figure 2. Forest Plot of the Mean Resilience Score, According to the Type of Disease

taking medicine and preserving his/her own therapeutic diet.

Resilience shows the way of healthier life to patients, so that they become more adapted to changes in their life and more eager to take part in treatment programs (31). Given that progress of the symptoms may cause negative effects on mental health and higher vulnerability to the disease in turn, resilience is an effective way to deal with the pressure caused by the disease (41). In the present study, the mean resilience score of the European participants was higher than that of the Asian, American, and Australian patients. This might be because the resilience tools were mostly designed for Western cultures. Protective factors are the needed specifications or situations to develop resilience that also adjust the effects of dealing with threats and improve outcomes of resilience (42). Taking into account that resilience reflects beliefs, attitudes, and ideology (43), it could be argued that protective factors differ according to the gender, race, and culture of the patient. Culture is effective on resilience so that a specific behavior that supports resilience in one culture may have negative effects on resilience in another factor. Ho highlighted dif- 


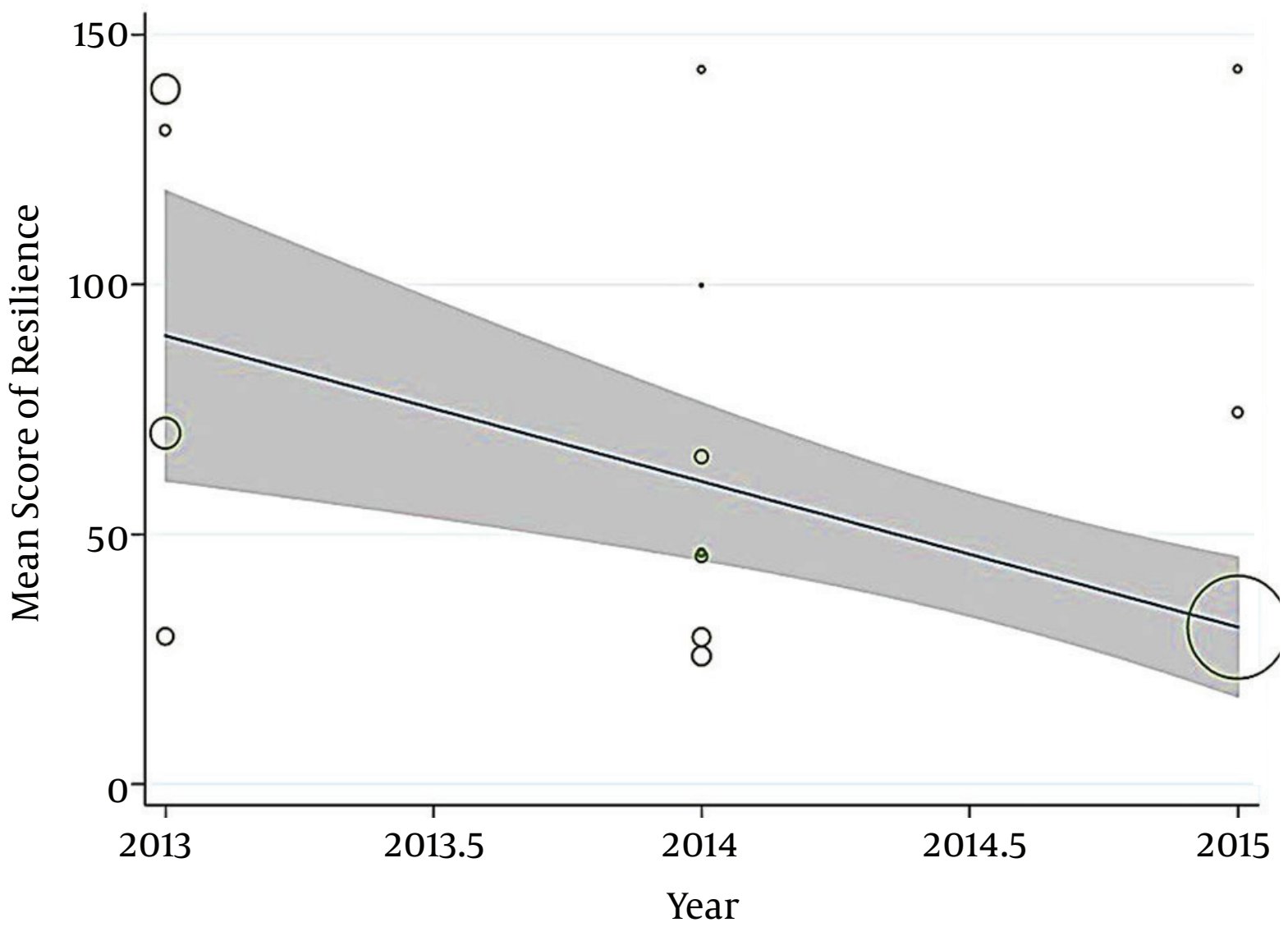

Figure 3. Meta-Regression Plot of the Mean Resilience Score, According to the Year of the Study

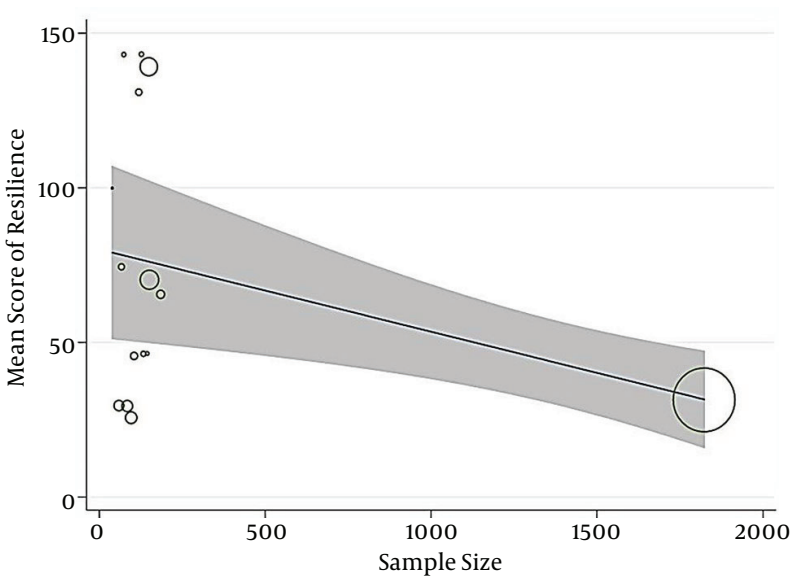

Figure 4. Meta-Regression Plot of the Mean Resilience Scores of the Chronic Diseases Patients, According to the Study Sample Size

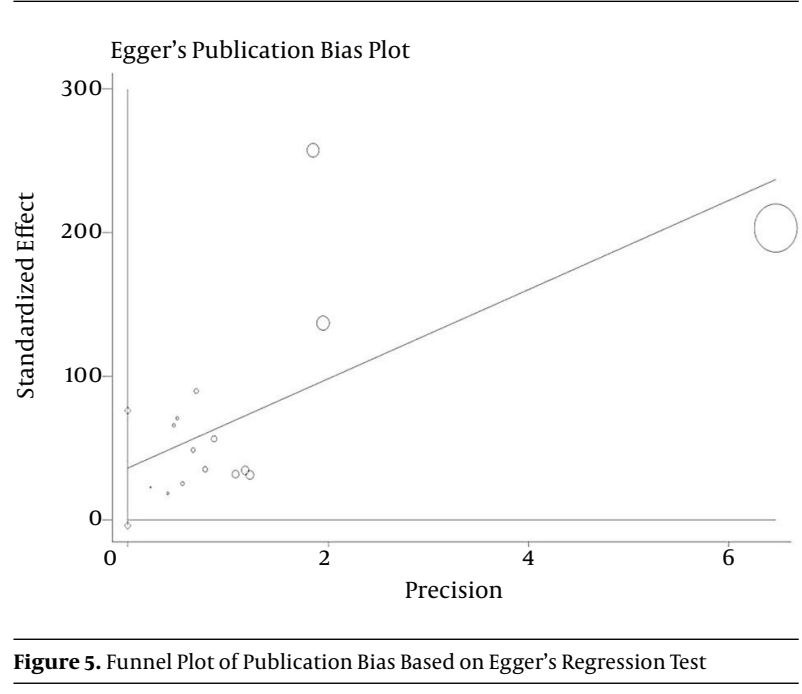

ferences in the attitudes of people in the West and East to resilience, with Asian cultures viewing resilience as aware- 
ness and acceptance of experience and Western cultures considering it as self-choice and mastery over the environment (44).

The results of the meta-regression showed that there was no relationship between the year of publication, sample size, and resilience. Thus, the number of participants or recent environmental and social changes, including wars, disasters, and technological events, did not seem to influence the resilience score. Given that the purpose of a systematic review is to accurately survey the literature, provide a quantitative summary of the results, combine the results, and conclude with a general interpretation of the results, our conclusion represents wider picture of the results of different studies, which is an advantage of the present study.

The strong point of the present article is that, unlike other studies, it focused on resilience exclusively in patients with chronic physical diseases. In a review study, Call et al. studied the resilience of patients with physical and psychological diseases (41). In another review study, Johnston et al. focused on resilience and its relation with the consequences (45). Hu et al. performed a meta-analysis of the relationship between resilience and psychological health (46). The main limitation of the present study was not having access to the full texts of some of the aforementioned studies. The findings of the present study point to the need to design a specific tool for patients with chronic physical diseases.

\section{Acknowledgments}

We sincerely thank all the researchers whose studies were used in the present meta-analysis; and also special thanks to the deputy for the university of social welfare and rehabilitation sciences for providing financial support and help for project.

\section{Footnotes}

Authors' Contribution: Reza Ghanei Gheshlagh and Abbas Ebadi, data collection; Kian Nourozi Tabrizi, study design; Asghar Dalvandi and Abbas Ebadi, final revision and grammar editing; Kourosh Sayehmiri and Sahar Dalvand, statistical analysis.

Financial Disclosure: The authors declare no conflict of interest.

Funding/Support: This study was supported by a grant from the deputies for research of the university of social welfare and rehabilitation sciences.

\section{References}

1. Compas BE, Connor-Smith JK, Saltzman H, Thomsen AH, Wadsworth ME. Coping with stress during childhood and adolescence: problems, progress, and potential in theory and research. Psychol Bull. 2001;127(1):87-127. [PubMed: 11271757].

2. Eggenberger SK, Meiers SJ, Krumwiede N, Bliesmer M, Earle P. Reintegration within families in the context of chronic illness: A family health promoting process. J Nurs Healthc Chronic Illn. 2011;3(3):283-92.

3. Livneh $\mathrm{H}$, Antonak RF. Psychosocial adaptation to chronic illness and disability: A primer for counselors. J Couns Dev. 2005;83(1):12-20.

4. de Ridder D, Geenen R, Kuijer R, van Middendorp H. Psychological adjustment to chronic disease. Lancet. 2008;372(9634):246-55. doi: 10.1016/S0140-6736(08)61078-8. [PubMed: 18640461].

5. Akhbardeh M. Role of spiritual beliefs and prayer in health promotion of chronic patients: A qualitative study. Quran Med. 2011;2011(1, Summer [En]):5-9.

6. Sauer PA. Does resilience mediate the effects of bullying in nurses? University of North Carolina at Greensboro; 2013.

7. Rosen JA, Glennie EJ, Dalton BW, Lennon JM, Bozick RN. Noncognitive skills in the classroom: New perspectives on educational research. ERIC; 2010

8. Basim HN, Cetin F. The reliability and validity of the resilience scale for adults. Turk Psikiyatri Derg. 2011;22(2):104-14.

9. Reis SM, Colbert RD, Hébert T. Understanding resilience in diverse, talented students in an urban high school. Roeper Rev. 2004;27(2):110-20.

10. Wagnild GM, Young HM. Development and psychometric evaluation of the Resilience Scale. J Nurs Meas. 1993;1(2):165-78. [PubMed: 7850498].

11. Garroway A. Resilience in Parkinson's disease: An empirical examination of age-related components of the construct. 2014

12. Tusaie K, Dyer J. Resilience: a historical review of the construct. Holist Nurs Pract. 2004;18(1):3-8. [PubMed:14765686] quiz 9-10.

13. Shakespeare-Finch J, Gow K, Smith S. Personality, coping and posttraumatic growth in emergency ambulance personnel. Traumatology. 2005;11(4):325.

14. Meichenbaum D, editor. Understanding resilience in children and adults: Implications for prevention and interventions. paper delivered to the Melissa Institute Ninth Annual Conference on Resilience. 2005; Citeseer.

15. McAllister M, McKinnon J. The importance of teaching and learning resilience in the health disciplines: a critical review of the literature. Nurse Educ Today. 2009;29(4):371-9. doi: 10.1016/j.nedt.2008.10.011. [PubMed: 19056153].

16. Sadeghi S, Maarefvand M, Shoja A. Relation of Creativity and Resiliency in Child Laborers. Social Work. 2014;3(1):10-5.

17. Santos F, Bernardo V, Gabbay M, Dib SA, Sigulem D. The impact of knowledge about diabetes, resilience and depression on glycemic control: a cross-sectional study among adolescents and young adults with type 1 diabetes. Diabetol Metab Syndr. 2013;5(1):1.

18. Steinhardt MA, Mamerow MM, Brown SA, Jolly CA. A resilience intervention in African American adults with type 2 diabetes: a pilot study of efficacy. Diabetes Educ. 2009;35(2):274-84. doi: 10.1177/0145721708329698. [PubMed: 19204102].

19. Kilic SA, Dorstyn DS, Guiver NG. Examining factors that contribute to the process of resilience following spinal cord injury. Spinal Cord. 2013;51(7):553-7. doi: 10.1038/sc.2013.25. [PubMed: 23689391].

20. Chan I, Lai J, Wong K. Resilience is associated with better recovery in Chinese people diagnosed with coronary heart disease. Psychology Health. 2006;21(3):335-49.

21. Robottom BJ, Gruber-Baldini AL, Anderson KE, Reich SG, Fishman PS, Weiner WJ, et al. What determines resilience in patients with Parkinson's disease?. Parkinsonism Relat Disord. 2012;18(2):174-7. doi 10.1016/j.parkreldis.2011.09.021. [PubMed: 21975263].

22. Ungar M. Handbook for working with children and youth: Pathways to resilience across cultures and contexts. Sage Publications; 2005. 
23. Schetter CD, Dolbier C. Resilience in the Context of Chronic Stress and Health in Adults. Soc Personal Psychol Compass. 2011;5(9):634-52. doi 10.1111/j.1751-9004.2011.00379.x. [PubMed: 26161137].

24. Windle G, Bennett KM, Noyes J. A methodological review of resilience measurement scales. Health Qual Life Outcomes. 2011;9:8. doi: 10.1186/1477-7525-9-8. [PubMed: 21294858].

25. Moher D, Liberati A, Tetzlaff J, Altman DG, Prisma Group. Preferred reporting items for systematic reviews and meta-analyses: the PRISMA statement. Ann Intern Med. 2009;151(4):264-9. [PubMed: 19622511] W64.

26. Tsimicalis A, Stinson J, Stevens B. Quality of life of children following bone marrow transplantation: critical review of the research literature. Eur J Oncol Nurs. 2005;9(3):218-38. doi: 10.1016/j.ejon.2004.08.006. [PubMed:16112524].

27. Dubey C, De Maria J, Hoeppli C, Betticher DC, Eicher M. Resilience and unmet supportive care needs in patients with cancer during early treatment: A descriptive study. Eur J Oncol Nurs. 2015;19(5):582-8. doi: 10.1016/j.ejon.2015.03.004. [PubMed: 25882547].

28. Liu JC, Chang LY, Wu SY, Tsai PS. Resilience mediates the relationship between depression and psychological health status in patients with heart failure: a cross-sectional study. Int J Nurs Stud. 2015;52(12):184653. doi: 10.1016/j.ijnurstu.2015.07.005. [PubMed: 26254853].

29. Rosenberg AR, Syrjala KL, Martin PJ, Flowers ME, Carpenter PA, Salit RB, et al. Resilience, health, and quality of life among longterm survivors of hematopoietic cell transplantation. Cancer 2015;121(23):4250-7. doi: 10.1002/cncr.29651. [PubMed: 26288023].

30. Arrebola-Moreno AL, Garcia-Retamero R, Catena A, Marfil-Álvarez R, Melgares-Moreno R, Ramírez-Hernández JA. On the protective effect of resilience in patients with acute coronary syndrome. Int J Clin Health Psychol. 2014;14(2):111.

31. Faria DA, Revoredo LS, Vilar MJ, Eulalia Maria Chaves M. Resilience and treatment adhesion in patients with systemic lupus erythematosus. Open Rheumatol J. 2014;8:1-8. doi: 10.2174/1874312920140127001. [PubMed: 24665352].

32. Hayter MR, Dorstyn DS. Resilience, self-esteem and self-compassion in adults with spina bifida. Spinal Cord. 2014;52(2):167-71. doi 10.1038/sc.2013.152. [PubMed: 24322213].

33. Schumacher A, Sauerland C, Silling G, Berdel WE, Stelljes M. Resilience in patients after allogeneic stem cell transplantation. Support Care Cancer. 2014;22(2):487-93. doi: 10.1007/s00520-013-2001-6. [PubMed:
24101151].

34. Dale SK, Cohen MH, Kelso GA, Cruise RC, Weber KM, Watson C, et al. Resilience among women with HIV: Impact of silencing the self and socioeconomic factors. Sex Roles. 2014;70(5-6):221-31. doi:10.1007/s11199014-0348-x. [PubMed: 24932061].

35. DoustdarTousi S, Golshani S. Effect of resilience in patients hospitalized with cardiovascular diseases. JMUMS. 2014;24(116).

36. Noori Saeid A, Akbari B, Salari A. Comparing the psychological resiliency in patients with coronary artery disease and normal subjects. J Guilan Univ Med Sci. 2014;23(91):47-52.

37. Amirpour B. Comparison of Resiliency and depression in cardiac patients and normal people. Razi J Med Sci. 2014;21(126):73-82.

38. Ma LC, Chang HJ, Liu YM, Hsieh HL, Lo L, Lin MY, et al. The relationship between health-promoting behaviors and resilience in patients with chronic kidney disease. ScientificWorldJournal. 2013;2013:124973. doi: 10.1155/2013/124973. [PubMed: 23589703].

39. Moe A, Ekker K, Enmarker I. A description of resilience for Norwegian home-living chronically ill oldest older persons. 2013

40. Min JA, Yoon S, Lee CU, Chae JH, Lee C, Song KY, et al. Psychological resilience contributes to low emotional distress in cancer patients. Support Care Cancer. 2013;21(9):2469-76. doi:10.1007/s00520-013-18076. [PubMed: 23604453].

41. Cal S, Sa L, Glustak M, Santiago M. Resilience in chronic diseases: a systematic review. Cogent Psychol. 2015;2(1):1024928.

42. Huang M. Resilience in chronic disease: the relationships among risk factors, protective factors, adaptive outcomes, and the level of resilience in adults with diabetes. 2009

43. Cheung C, Kam P. Resiliency in older Hong Kong Chinese: Using the grounded theory approach to reveal social and spiritual conditions.J Aging Stud. 2012;26(3):355-67.

44. He J, Van de Vijver F. Youth resilience and culture. Springer; 2015. pp. 189-201.The value of keeping an open eye for methodological issues in research on resilience and culture.

45. Johnston MC, Porteous T, Crilly MA, Burton CD, Elliott A, Iversen $\mathrm{L}$, et al. Physical disease and resilient outcomes: a systematic review of resilience definitions and study methods. Psychosomatics. 2015;56(2):168-80. doi: 10.1016/j.psym.2014.10.005. [PubMed: 25620566].

46. Hu T, Zhang D, Wang J.A meta-analysis of the trait resilience and mental health. Pers Individ Dif. 2015;76:18-27. 